\title{
EXISTENCE OF FINITE INVARIANT MEASURES FOR MARKOV PROCESSES
}

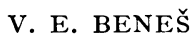

Let $x_{t}$ be a Markov process on a locally compact metric space $(\varepsilon, \rho)$ with a countable base. Let $\beta$ be the $\sigma$-algebra generated by the open sets, and for $A \in B, t>0$, set $P(t, x, A)=\operatorname{Pr}\left\{x_{t} \in A \mid x_{0}=x\right\}$. Khasminskii [1] has related, in a natural way, the existence of a finite invariant measure for $x_{t}$ with the mean time to hit a compact set. Our object is to relate the existence of such a measure to properties of the measures $P(t, x, \cdot), t>0, x \in \mathcal{E}$.

We assume that for fixed $t$ and $A, P(t, x, A)$ is continuous in $x$, that for an $\epsilon$-neighborhood $S_{\epsilon}(x)$ about $x, P\left(t, x, S_{\epsilon}(x)\right) \rightarrow 1$ as $t \rightarrow 0$ uniformly on compacta, and that $P(t, x, \varepsilon)=1$. Let $C^{+}$be the strictly positive cone of the Banach space ca $(\mathcal{E}, \mathbb{B})$ consisting of the countably additive set functions on $B$ with variation norm. Let $U_{t}, t \geqq 0$, be the semigroup defined for $\mu \in \mathrm{ca}(\mathcal{E}, B)$ by the formula

$$
U_{t} \mu(A)=\int_{\mathcal{E}} P(t, x, A) \mu(d x),
$$

and let $(f, \mu)$ denote the action of a linear functional on $\mu \in \mathrm{ca}(\mathcal{E},()$. A B-measurable function $f(\cdot)$ on $\mathcal{E}$ is a moment iff $f \geqq 0$ and $\inf _{x \in \mathcal{E}-K_{n}} f(x) \rightarrow \infty$ as some sequence of compact $K_{n} \uparrow \mathcal{E}$.

THEOREM. The following conditions are equivalent:

(i) $x_{t}$ has a positive finite invariant measure;

(ii) $\exists \mu \in C^{+}, \exists$ moment $g$, with $\sup _{t \geq 0}\left(g, U_{t} \mu\right)<\infty$;

(iii) $\exists \mu \in C^{+}$such that the measures $U_{t} \mu, t \geqq 0$, are uniformly countably additive;

(iv) $\exists \mu, \nu \in C^{+}$such that the measures $U_{t} \mu, t \geqq 0$ are uniformly $\nu$-continuous;

(v) $\exists \mu \in C^{+}, \exists K$ weakly compact in $\mathrm{ca}(\mathcal{E}, \Theta), K$ contains the orbit $\left\{U_{t} \mu, t \geqq 0\right\}$.

Lemma 1. For $K$ compact,

$$
\lim _{h \downarrow 0} \sup _{y \in K}|P(t+h, y, A)-P(t, y, A)|=0 .
$$

Proof. Pick $r>0$ so that $U=S_{r}(K)=\{y: \rho(y, K)<r\}$ has compact closure. Then $P(h, y, U) \geq P\left(h, y, S_{r}(y)\right)$, and $P(h, y, U) \rightarrow 1$ as $h \downarrow 0$, uniformly on $K$. We write for any $\delta>0$

Presented to the Society, August 31, 1966; received by the editors October 4, 1966. 


$$
\begin{aligned}
P(t & +h, y, A)-P(t, y, A) \\
& =\left\{\int_{U \cap S_{\delta}(y)}+\int_{\left.{\mathcal{E}-\left(U \cap S_{\delta}(y)\right)}\right\}}[P(t, w, A)-P(t, y, A)] P(h, y, d w) .\right.
\end{aligned}
$$

Let $\epsilon>0$ be given. The closure of $U$ is compact, so it is possible to pick $\delta>0$ so small that $w, z \in U, \rho(w, z) \leqq \delta$ imply $|P(t, w, A)-P(t, z, A)|$ $\leqq \epsilon / 2$; with this choice of $\delta$ the first integral is at most $\epsilon / 2$ in magnitude. The second integral is in magnitude at most

$$
1-P\left(h, y, S_{\delta}(y)\right)-P(h, y, U)+P\left(h, y, S_{\delta}(y) \cup U\right),
$$

which tends to 0 as $h \downarrow 0$ uniformly on $K$.

Lemma 2. For $t>0, K$ compact,

$$
\lim _{h \downarrow 0} \sup _{y \in K}|P(t-h, y, A)-P(t, y, A)|=0 .
$$

Proof. Let $\epsilon>0$ be given. Choose $r>0$ so that $S_{r}(K)$ has compact closure and then $\delta<t$ so small that $s \leqq \delta$ implies

$$
\sup _{y \in K} P\left(s, y, \mathcal{E}-S_{r}(K)\right) \leqq \epsilon / 2 .
$$

Considering only $0 \leqq h \leqq \delta$, we write

$$
\begin{aligned}
& P(t-h, y, A)-P(t, y, A)=\left\{\int_{S_{r}(K)}=\int_{\mathcal{E}_{-S_{r}(K)}}\right\} \\
& \cdot[P(t-\delta, z, A)-P(t-\delta+h, z, A)] P(\delta-h, y, d z) .
\end{aligned}
$$

By Lemma 1 choose $s \leqq \delta$ so small that $h \leqq s$ implies

$$
\sup _{z \in S_{r}(K)}|P(t-\delta, z, A)-P(t-\delta+h, z, A)| \leqq \epsilon / 2 .
$$

Lemma 3. For fixed $A \in \Theta, P(t, y, A)$ is $(t, y)$-continuous in the product topology of $[0, \infty) \times \varepsilon$.

Proof. Let $\epsilon>0$ be given. Writing, with $h>0$ if $t=0$,

$$
\begin{aligned}
P(t, y, A)- & P(t+h, z, A) \\
& =P(t, y, A)-P(t, z, A)+P(t, z, A)-P(t+h, z, A),
\end{aligned}
$$

we pick by Lemmas 1 and 2, and by the z-continuity of $P(t, z, A)$, a neighborhood $U$ of $y$ with compact closure and a number $s>0$ such that $|h|<s$ implies 


$$
\sup _{z \in U}|P(t, z, A)-P(t+h, z, A)|<\epsilon / 2
$$

and

$$
\sup _{z \in U}|P(t, y, A)-P(t, z, A)|<\epsilon / 2 .
$$

Proof of Theorem. We show (i) $\Rightarrow$ (ii) $\Rightarrow$ (iii) $\Rightarrow$ (iv) $\Rightarrow$ (v) $\Rightarrow$ (i).

(i) $\Rightarrow$ (ii): $\varepsilon$ is $\sigma$-compact. If $\nu$ is finite and invariant, choose $K_{n}$ compact $\uparrow \mathcal{E}$. If $a_{k}, k \geqq 1$ is a nonnegative summable sequence, then there exists a nonnegative sequence $b_{k}, k \geqq 1$ with $b_{k} \rightarrow \infty$ as $k \rightarrow \infty$ and $\sum_{n=1}^{\infty} a_{n} b_{n}<\infty$. Set $a_{n}=\nu\left(K_{n+1}-K_{n}\right), g(x)=b_{n}$ for $x \in K_{n+1}-K_{n}$. Then $g$ is a moment and $\left(g, U_{t} \nu\right) \equiv$ constant for $t \geqq 0$.

(ii) $\Rightarrow$ (iii): By Lemma 1 and Dini's theorem [2, p. 239], $P(t, x, A) \rightarrow 0$ as $A \downarrow \phi$, uniformly on $(t, x)$-compacta. Let $s>0$ be fixed, $K_{n}$ compact $\uparrow \varepsilon$. For $t \leqq s$,

$$
U_{t} \mu(A) \leqq \sup _{y \in K_{n}} P(t, y, A) \mu\left(K_{n}\right)+\mu\left(\mathcal{E}-K_{n}\right) .
$$

For $t>s$, with $c=\sup _{t \geqq 0}\left(g, U_{t} \mu\right)$,

$$
U_{t} \mu(A) \leqq \sup _{y \in K_{n}} P(s, y, A) \mu(\mathcal{E})+c / \inf _{x \in \mathcal{E}-K_{n}} g(x) .
$$

With $\epsilon>0$ given, choose first $n$ so large that

$$
\mu\left(\mathcal{E}-K_{n}\right) \leqq c / \inf _{x \in \mathcal{E}-K_{n}} g(x)<\epsilon / 2,
$$

and then choose $B \in ß$ so near $\phi$ that $A \subseteq B$ implies

$$
\mu(\varepsilon) \sup _{0 \leqq h \leqq s} \sup _{y \in K_{n}} P(h, y, A)<\epsilon / 2 .
$$

Hence $\lim _{A \downarrow \phi} \sup _{t \geq 0} U_{t} \mu(A)=0$, and this implies uniform countable additivity.

(iii) $\Rightarrow$ (iv) $\Rightarrow(\mathrm{v})$ : By $\left[3\right.$, Theorem 1, p. 305], the orbit $\left\{U_{t} \mu, t \geqq 0\right\}$ is weakly sequentially compact. Uniform absolute continuity w.r. to a fixed finite positive measure follows from [3, Theorem 2, p. 306]. By the Eberlein-Šmulyan theorem [3, p. 430] the weak closure of the orbit is weakly compact.

$(\mathrm{v}) \Rightarrow(\mathrm{i})$ : Each $U_{t}$ is weakly continuous, since for $f \in \mathrm{ca}(\varepsilon, \Theta)^{*}$, $\left(f, U_{t} \cdot\right)$ is just another continuous linear functional. Let $O$ be the orbit $\left\{U_{t} \mu, t \geqq 0\right\}$, and let co $O$ and clco $O$ be its convex and closed convex hull, respectively (weak topology). Clearly clco $O \subseteq$ clco $K$. Since $K$ is weakly compact, the Krein-Smulyan theorem implies that clco $K$ is itself weakly compact. Since clco $O$ is a closed subset of 
clco $K$, it too is weakly compact. If now $\nu \in \operatorname{clco} O$, there exists a generalized sequence $\nu_{\alpha} \in \operatorname{co} O$ with $\nu_{\alpha} \rightarrow \nu$ weakly. Thus $U_{t} \nu_{\alpha} \in \operatorname{co} O$, and $U_{t} \nu_{\alpha} \rightarrow U_{t} \nu$ weakly. Hence each $U_{t}$ carries clco $O$ in to itself. Evidently $U_{t} U_{s}=U_{s} U_{t}$. Thus by the Markov-Kakutani fixed-point theorem $\left[3\right.$, p. 456], there exists a measure $\nu \in \operatorname{clco} O$ with $U_{t} \nu=\nu$ for all $t \geqq 0$. Since $\mu>0$, the orbit $O$ is confined to a sphere bounded away from the origin and $\nu$ can be normalized as a probability measure.

To see this, we use a result of Mazur [3, p. 422] to the effect that cl co $O$ is closed in the norm topology. Then $\nu$ is a strong limit point of elements in co $O$. There is no restriction in taking $\|\mu\|=1$ so that co $O$ consists entirely of probability measures. We will be able to find a sequence $\nu_{n} \in$ co $O$ with $\left\|\nu_{n}-\nu\right\| \rightarrow 0$ (sequences are adequate since strong topology is metric). Since $\left\|\nu_{n}\right\|=1$, it follows that $\|\nu\|=1$.

S. P. Lloyd has remarked that $\nu$ will be unique within clco $O$. Let $\zeta \in$ clco $O$ be invariant. By Mazur's theorem there exist sequences $\nu_{n} \rightarrow \nu, \zeta_{m} \rightarrow \zeta$ in norm with $\nu_{n}, \zeta_{m} \in \operatorname{co} O$, i.e., $\nu_{n}=P_{n} \mu, \zeta_{m}=P_{m} \mu$ for convex combinations $P_{n}, P_{m}$ of operators $U_{t}$. Clearly $P_{m} \nu=\nu$, $P_{n} \zeta=\zeta, P_{n} P_{m}=P_{m} P_{n}$. Thus

$$
\|\nu-\zeta\|=\left\|P_{m} \nu-P_{n} \zeta\right\|=\left\|P_{m}\left(\nu-P_{n} \mu\right)+P_{n}\left(P_{m} \mu-\zeta\right)\right\| .
$$

\section{REFERENCES}

1. R. Z. Khasminskii, Ergodic properties of recurrent diffusion processes and stabilization of the solution to the Cauchy problem for parabolic equations, Theor. Probability Appl. 5 (1960), 179-196.

2. J. L. Kelley, General topology, Van Nostrand, New York, 1955.

3. N. Dunford and J. T. Schwartz, Linear operators, Part I, Interscience, New York, 1958.

Bell Telephone Laboratories, Incorporated 